\title{
Localization and Mobility Edge in One-Dimensional Potentials with Correlated Disorder
}

\author{
F.M. Izrailev and A.A. Krokhin \\ Instituto de Física, Universidad Autonoma de Puebla, Apdo. Postal J-48, Puebla, 72570 México
}

(August 7, 2018)

\begin{abstract}
We show that a mobility edge exists in $1 \mathrm{D}$ random potentials provided specific long-range correlations. Our approach is based on the relation between binary correlator of a site potential and the localization length. We give the algorithm to construct numerically potentials with mobility edge at any given energy inside allowed zone. Another natural way to generate such potentials is to use chaotic trajectories of non-linear maps. Our numerical calculations for few particular potentials demonstrate the presence of mobility edges in 1D geometry.
\end{abstract}

PACS numbers: 72.15.Rw, 03.65.BZ, 72.10.Bg

It is commonly believed that there is no mobility edge in 1D models with random-like potentials. This is based on the fact that for random potentials all eigenstates are exponentially localized, no matter how weak the randomness is [1]. On the other hand, for potentials with "correlated disorder" the localization length diverges for specific values of energy (see, e.g. 22,3]). The well-studied model of this kind is the so-called "random dimer" 四 for which the potential has peculiar short-range correlations. Though there is no mobility edge for such potentials, this example shows a highly non-trivial role of correlations. In this Letter we study the relation between correlations in the site-potential of 1D tight-binding model and localization properties of eigenstates, and give examples of the potentials with mobility edges inside the energy band.

The model under consideration is the discrete Shrödinger equation for stationary eigenstates $\psi_{n}(E)$,

$$
\psi_{n+1}+\psi_{n-1}=\left(E+\epsilon_{n}\right) \psi_{n},
$$

where $E$ is the eigenenergy and $\epsilon_{n}$ is the site-potential. To study the origin of delocalized states in longcorrelated random potentials, we suggest a simple and clear approach based on the representation of the quantum model (11) in terms of classical two-dimensional Hamiltonian map,

$$
\begin{gathered}
p_{n+1}=p_{n}+\left(E-2-\epsilon_{n}\right) x_{n}, \\
x_{n+1}=x_{n}+p_{n+1},
\end{gathered}
$$

where $p_{n+1}=x_{n+1}-x_{n}$ and $x_{n}=\psi_{n}$. This map describes the behavior of a linear oscillator subjected to linear periodic delta-kicks with amplitude depending on $\epsilon_{n}$. In such an approach, localized quantum states correspond to trajectories which are unbounded in the classical phase space $(p, x)$ when $n \rightarrow \infty$. Contrary, extended states are represented by bounded trajectories.

It is convenient to introduce the action-angle variables $(r, \theta)$ and represent Eqs.(11) and (2) in the followind form (see details in 河),

$$
\begin{aligned}
\sin \theta_{n+1} & =D_{n}^{-1}\left(\sin \left(\theta_{n}-\mu\right)-A_{n} \sin \theta_{n} \sin \mu\right), \\
\cos \theta_{n+1} & =D_{n}^{-1}\left(\cos \left(\theta_{n}-\mu\right)+A_{n} \sin \theta_{n} \cos \mu\right),
\end{aligned}
$$

where

$$
\begin{gathered}
D_{n}=\frac{r_{n+1}}{r_{n}}=\sqrt{1+A_{n} \sin \left(2 \theta_{n}\right)+A_{n}^{2} \sin ^{2} \theta_{n}}, \\
A_{n}=-\epsilon_{n} / \sin \mu, \quad E=2 \cos \mu .
\end{gathered}
$$

We use the following definition of the inverse localization length (or Lyapunov exponent $\Lambda$ ) 河],

$$
l^{-1} \equiv \Lambda=\lim _{N \rightarrow \infty} \frac{1}{N} \sum_{n=1}^{N} \ln \left(\frac{r_{n+1}}{r_{n}}\right),
$$

which coincides inside the energy band, $|E|<2$ with the standard definition [6], $\Lambda=\left\langle\ln \left|\frac{\psi_{n+1}}{\psi_{n}}\right|\right\rangle$ (see details in [7]). Here the brackets stand for the average over $n$. This Hamiltonian map approach turns out to be very effective in the study of completely disordered potentials [7] as well as potentials with correlated disorder [5,8].

Below we consider a general case of any stationary sitepotential $\epsilon_{n}$ under the condition $\left|\epsilon_{n}\right|<<1$. In this case one can expand the logarithm in Eq. (5) and in the second order of perturbation theory gets,

$$
l^{-1}=\frac{\left\langle\epsilon_{n}^{2}\right\rangle}{8 \sin ^{2} \mu}-\frac{\left\langle\epsilon_{n} \sin \left(2 \theta_{n}\right)\right\rangle}{2 \sin \mu} .
$$

In order to calculate the correlator $\left\langle\epsilon_{n} \sin \left(2 \theta_{n}\right)\right\rangle$ with quadratic accuracy, we use the approximate onedimensional map for the phase $\theta_{n}$ obtained from Eq. (3),

$$
\theta_{n}=\theta_{n-1}-\mu+\epsilon_{n-1} \frac{\sin ^{2} \theta_{n-1}}{\sin \mu} .
$$

From Eq. (7] the correlator $\left\langle\epsilon_{n} \sin \left(2 \theta_{n}\right)\right\rangle$ can be expressed through the precedent one, $\left\langle\epsilon_{n} \sin 2\left(\theta_{n-1}\right)\right\rangle$. Let us introduce the following notations,

$$
\begin{gathered}
Q_{k} \equiv\left\langle\epsilon_{n} e^{2 i \theta_{n-k}}\right\rangle=-\frac{i}{2} \frac{\exp (-2 i \mu)}{\sin \mu} \epsilon_{0}^{2} a_{k}, \\
\left\langle\epsilon_{n} \epsilon_{n-k}\right\rangle=\epsilon_{0}^{2} \xi(k), \quad\left\langle\epsilon_{n}^{2}\right\rangle=\epsilon_{0}^{2} .
\end{gathered}
$$


It can be shown that the normalized correlators $a_{k}$ are defined by the infinite set of linear equations,

$$
a_{k-1}-e^{-2 i \mu} a_{k}=\xi(k), \quad 1 \leq k<\infty .
$$

These equations emerge after multiple application of the recursion relation (7) to the correlator $Q_{k}$.

According to Eq. (6) the localization length is determined by the only term $a_{0}$,

$$
l^{-1}=\frac{\epsilon_{0}^{2}}{8 \sin ^{2} \mu}+\frac{\epsilon_{0}^{2}}{4 \sin ^{2} \mu} \operatorname{Re}\left(e^{-2 i \mu} a_{0}\right),
$$

which can be easily obtained from Eq. (9),

$$
a_{0}=\sum_{k=1}^{\infty} \xi(k) \exp [-2 i \mu(k-1)] .
$$

As a result, we come to the final expression for the inverse localization length,

$$
l^{-1}=\frac{\epsilon_{0}^{2} \varphi(\mu)}{8 \sin ^{2} \mu} ; \quad \varphi(\mu)=1+2 \sum_{k=1}^{\infty} \xi(k) \cos (2 \mu k) .
$$

Here, the function $\varphi(\mu)$ is given by the Fourier series with the coefficients $\xi(k)$ which are the correlators of the site potential $\epsilon_{n}$. Note that Eq. (12) fails close to the band center, $E=0$, and to the band edges, $E= \pm 2$, where even the standard Anderson model exhibits peculiarities (see, for example, [7] and references therein).

Eq. (12) establishes the relation between the localization length and correlations. It allows us to calculate the localization length if statistical properties of the sequence $\epsilon_{n}$ are known. Note that only binary correlators enter in Eq. (12). This property comes from the Born approximation and not from restrictions for statistics of $\epsilon_{n}$ [9]. In particular, we do not assume that the statistics is gaussian.

Formally, the same result has been derived by different methods in [9,10]. For continuous models, the highenergy asymptotics of the Lyapunov exponent is also determined by the correlation function of the site-potential [6]. To the best of our knowledge, the relation (12) has not been discussed yet concerning the problem of mobility edge. The advantage of the above approach based on the Hamiltonian representation, is that it allows a simple generalization to the Kronig-Penney model with correlated disorder [11] and to the models with off-diagonal disorder. The approach also clarifies the physical meaning of the summation in Eq.(12). Namely, the localization appears as a result of multiple scattering at different sites, see Eq.(3)), and each scattering enters in the Born approximation, see Eq.(7).

To reveal the role of correlations, let us apply the general relation (12) to some known models. First, we consider the potential

$$
\epsilon_{n}=\epsilon_{0} \sqrt{2} \cos \left(2 \pi \alpha n^{\gamma}\right)
$$

with $\alpha$ irrational. The correlation function $\xi(k)$ for this sequence is given by the sum

$$
\xi(k)=\lim _{N \rightarrow \infty} \frac{1}{N} \sum_{n=1}^{N} \cos \left(2 \pi \gamma \alpha k n^{\gamma-1}\right) .
$$

For $0<\gamma<1$ all the correlators $\xi(k)$ are independent on $k$ and equal 1 , thus, giving $\varphi(\mu)=0$ for $0<\mu<\pi$. Therefore, for this case the result coincides with the case of the constant potential $\epsilon_{n}=\epsilon_{0}$; in both cases the inverse localization length vanishes. This is consistent with the perturbative approach developed in 10].

For $\gamma=1$ Eq. (1) is reduced to the Harper equation with incommensurate potential. In this case the correlation function $\xi(k)$ oscillates, $\xi(k)=\cos (2 \pi \alpha k)$, that also gives $\varphi(\mu)=0$. Thus, in the Harper model with a weak potential all states are extended [12,13. For $\gamma>1$ the correlators $\xi(k)$ vanish and the localization length turns out to be the same as for the Anderson model (for energy close to the band center, see [14]).

Let us consider now the random dimer model [4] which is specified by the sequence $\epsilon_{n}$ having only two values $\epsilon_{1}$ and $\epsilon_{2}$, each of them appears in pairs $\left(\epsilon_{1} \epsilon_{1}\right.$ or $\left.\epsilon_{2} \epsilon_{2}\right)$ and each pair emerges with probability $1 / 2$. Fully transparent states are known to occur for $E=\epsilon_{1}$ and $E=\epsilon_{2}$, see, for example, [5] and references therein. Statistical properties of this model are characterized by the variance $\epsilon_{0}^{2}=\frac{1}{2}\left(\epsilon_{1}^{2}+\epsilon_{2}^{2}\right)$ and two correlators, $\left\langle\epsilon_{n} \epsilon_{n-1}\right\rangle=$ $\epsilon_{0}^{2} \frac{3+2 \lambda+3 \lambda^{2}}{4\left(1+\lambda^{2}\right)}$ and $\left\langle\epsilon_{n} \epsilon_{n-2}\right\rangle=\epsilon_{0}^{2} \frac{(1+\lambda)^{2}}{2\left(1+\lambda^{2}\right)}$ where $\lambda=\epsilon_{1} / \epsilon_{2}$. By substituting these correlators into Eq. 12, we obtain the inverse localization length for the dimer,

$$
l^{-1}(E)=\frac{\epsilon_{0}^{2}(1-\lambda)^{2}}{8\left(1+\lambda^{2}\right)} \frac{E^{2}}{4-E^{2}} .
$$

The generalization for $N$-mer when the values $\epsilon_{1}$ and $\epsilon_{2}$ appear in randomly distributed blocks of length $N$, can be readily done. For example, for the trimer we have:

$$
l^{-1}(E)=\frac{\epsilon_{0}^{2}(1-\lambda)^{2}}{12\left(1+\lambda^{2}\right)} \frac{\left(E^{2}-1\right)^{2}}{4-E^{2}} .
$$

Note that the above explicit expressions for dimer (15) and trimer (16) are given for the whole energy range; to the best of our knowledge, this is a new result.

For the $N$-mer constructed from the standard Anderson model by repeating $N$ times each random value $\epsilon_{n}$, Eq.(12) gives the same result obtained in [15]. In particular, this result shows that the correlations do not necessarily suppress the localization, they can make it even stronger than in the Anderson model [16].

The localization length (12) does not depend explicitly on the site-potential but on the binary correlators $\xi(k)$ of the potential. Let us consider different types of 
correlations. First, we take a sequence $\epsilon_{n}$ with exponential decay of correlations, $\xi(k)=\exp (-\beta k)$. This type of correlations is known to occur in completely chaotic Hamiltonian models without stable regions in the classical phase space. By substituting $\xi(k)$ into Eq. (12), one gets the following expression,

$$
l^{-1}=\frac{\epsilon_{0}^{2}}{8 \sin ^{2} \mu} \frac{\sinh \beta}{\cosh \beta-\cos (2 \mu)},
$$

which establishes a link between the correlation radius (parameter $\beta^{-1}$ ) of the site-potential, and the localization length of eigenstates. In the limiting case $\beta \rightarrow \infty$, we get the result for uncorrelated sequence (Anderson model) and for $\beta \rightarrow 0$ the result for the constant potential is recovered.

Concerning the power decay of correlations, one should note that such simple functions as $\xi(k)=1 / k$ or $1 / k^{2}$ can not serve as the correlation functions of any random sequence. Indeed, these functions lead to negative values of the Lyapunov exponent and do not satisfy the condition required for any correlation function [17. Specifically, the matrix $\xi_{k k^{\prime}} \equiv \xi\left(k-k^{\prime}\right)$ must be positive-semidefinite, the property which is not obvious a priori for arbitrary positive function $\xi(k)$.

An important application of Eq. (12) is related to the question about existence of the mobility edge in $1 \mathrm{D}$ systems. So far the mobility edge has been predicted for incommensurate potentials Eq.(13) with $\gamma<1$ and finite amplitude $\epsilon_{0}$ [18,19, and for Kronig-Penney model with constant electric field [20].

The relation (12) allows us to construct site potentials with mobility edges. Rewritten in the form

$$
\xi(k)=\frac{2}{\pi} \int_{0}^{\pi / 2} \varphi(\mu) \cos (2 k \mu) d \mu
$$

this relation gives a solution of the "inverse problem", namely, it shows how to calculate the correlation function $\xi(k)$ of the random potential if the normalized Lyapunov exponent $\Lambda_{0}(E)=\frac{2}{3} \varphi(\arccos (E / 2))$ is known. In other words, for any dependence $\Lambda_{0}$ there exist a set of sequences $\epsilon_{n}$ with correlation function $\xi(k)$.

To reconstruct the sequence via the correlation function, we have used the following algorithm [21],

$$
\epsilon_{n}=A \sum_{k=-\infty}^{+\infty} \xi(k) Z_{n+k}
$$

where $A$ is the normalization constant and $Z_{s}$ are random numbers from the interval $[0,1]$. As an example, we consider two sequences $\epsilon_{n}$ which exhibit the mobility edges at $E= \pm 1$. The first one was obtained from Eq.(19) by substituting $\xi(k)=\frac{3}{2 \pi k} \sin \left(\frac{2 \pi k}{3}\right)$ and $A=1$. This corresponds to the step-function dependence of the
Lyapunov exponent, $\Lambda_{0}=0$ for $|E|<1$ and $\Lambda_{0}=1$ for $1<|E|<2$. Circles in Fig. 1 show the dependence $\Lambda_{0}(E)$ calculated numerically for the reconstructed sequence $\epsilon_{n}$ using Eq. (5). The step-function is reproduced quite well and oscillations at $|E|>1$ are mainly due to the finite length of the sequence, $N=10^{6}$.

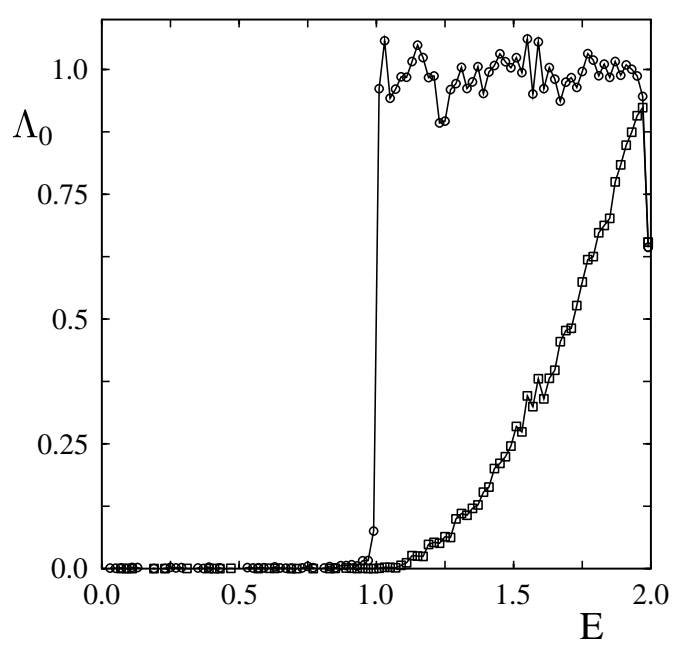

FIG. 1. Rescaled Lyapunov exponent $\Lambda_{0}(E)$ for two site-potentials $\epsilon_{n}$ constructed by Eq. (19) for 300 correlators $\xi(k)=\xi(-k)$ and $n=1, \ldots, 10^{6}$.

Another sequence $\epsilon_{n}$ was generated in order to get a smooth increase $\Lambda_{0}(E)$ for $|E| \geq 1$, see squares in Fig. 1. To do this, we used the correlators $\xi(k)$ which are the Fourier components (18) of the linear function $\Lambda_{0}(E)=1.53(|E|-1)$ for $|E| \geq 1$. As one can see, actual dependence $\Lambda_{0}(E)$ in Fig. 1 for $E>1$ clearly differs from the linear one. This results from the fact that in general case the algorithm (19) reconstructs the sequence $\epsilon_{n}$ with correlations $\xi(k)$ only asymptotically, for $k \gg 1$ 21. Nevertheless, it allows us to generate sequences with different values of critical exponents at the mobility edge. These examples demonstrate the existence of mobility edges for 1D site potentials with long-range correlations.

Another way to obtain explicitly the sequence $\epsilon_{n}$ with a mobility edge is to use weakly chaotic non-linear maps. Let us take the well-known standard map 22],

$$
\begin{gathered}
P_{n+1}=P_{n}+K \sin 2 \pi X_{n} \quad(\bmod 1) \\
X_{n+1}=X_{n}+P_{n+1} \quad(\bmod 1)
\end{gathered}
$$

For $K<1$ there are trajectories close to separatrices of non-linear resonance, with a very slow decay of correlations. For one of such trajectories (see the inset in Fig. 2) we have constructed the sequence $\epsilon_{n}$ as follows, $\epsilon_{n}=2 \epsilon_{0} \sin \left(2 \pi X_{n}\right)$, and calculated the Lyapunov exponent $\Lambda_{0}(E)=8 \Lambda \sin ^{2} \mu / \epsilon_{0}^{2}$ from Eq.(5). The dependence given in Fig. 2 for $\epsilon_{0}=0.1$ clearly indicates the presence of two mobility edges. It is important that the mobility edges are stable with respect to rather wide variations of 
$\epsilon_{0}$. Independent computation of eigenenergies of Eq. (11) with the corresponding potential $\epsilon_{n}$ demonstrates that the energy region shown in Fig. 2, indeed, belongs to the energy spectrum.

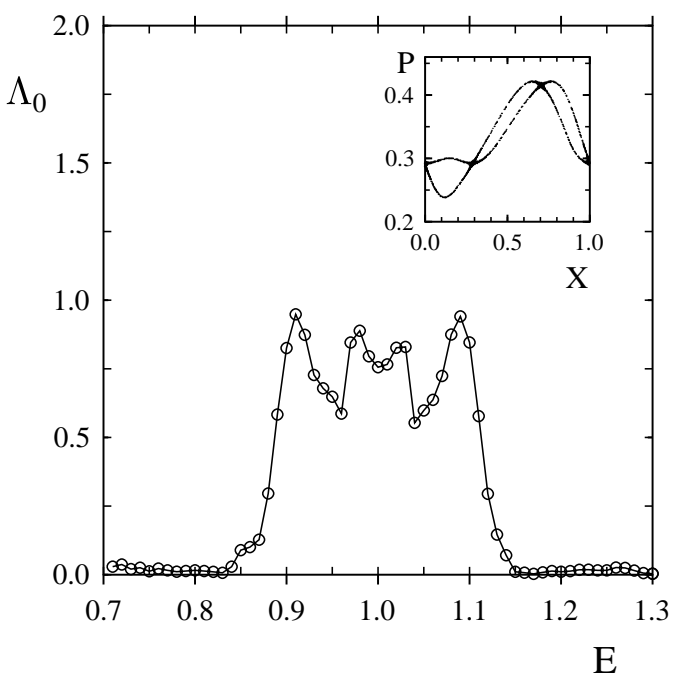

FIG. 2. Dependence $\Lambda_{0}(E)$ for the model (2) with the site potential defined by Eq.(20). The length of the sequence $\epsilon_{n}$ is $N=10^{6}$. The corresponding trajectory $\left(X_{n}, P_{n}\right)$ for $X_{0}=0, P_{0}=0.292$ and $K=0.8$ is shown in the inset.

It is interesting to note that inside a narrow chaotic region like that shown in the inset of Fig. 2, the time dependence of trajectory $\left(X_{n}, P_{n}\right)$ is given by regular rotation around the resonance of period 3 with the following chaotic motion in the vicinities of the crossed separatrices. This type of motion can be compared to the so-called intermittency which is well studied in 1D maps. Therefore, one can expect that there is a direct link between the correlations in the site potential and the intermittency in the evolution of the corresponding dynamical model which generates this potential (see also 24).

In conclusion, we have shown the existence of the mobility edge in $1 \mathrm{D}$ random potentials. The relation (12) allows us to determine binary correlation function of the site potential which exhibits any desirable dependence of the localization length on energy. Specific problem we address in this Letter, is how to reconstruct the potential itself from the binary correlations. We suggest an algorithm to calculate it numerically, and present the data for few specific potentials, with two mobility edges inside the allowed energy zone. Potentials exhibiting mobility edges can be also constructed by making use of weakly chaotic trajectories of non-linear maps, this is demonstrated using the standard map. Although analytical results are derived in quadratic approximation with random potential, our numerical data clearly show that the mobility edges do not disappear for relatively strong potentials. This fact opens a possibility for customer design of superlattices with anomalous electronic transport, in particular, devices with selective transparancy.

Very recently, the so-called self-similar potentials with specific long-range correlations have been studied [25], for which numerical data cleraly indicate the exictence of mobility edges close to the center of the enery band.

We thank J. Bellissard, S. Gredeskul, I. Guarneri, M. Hilke, I. Satija and V. Sokolov for useful discussions. This work was supported by CONACyT (Mexico) Grants No. 26163-E and No.28626-E.

[1] P. W. Anderson, Phys. Rev. 109, 1492 (1958).

[2] J.C. Flores, J.Phys. Condens. Matter 1, 8471 (1989).

[3] A. Bovier, J.Phys. A: Math. Gen., 25, 1021 (1992).

[4] D. Dunlap, H.-L. Wu, and P. Phillips, Phys. Rev. Let. 65, 88 (1990); P. Phillips and H.-L. Wu, Science 252, 1805 (1991).

[5] F.M.Izrailev, T.Kottos, and G.P.Tsironis, Phys. Rev. B. 52, 3274 (1995).

[6] I.M. Lifshitz, S. Gredeskul, and L. Pastur, Introduction to the Theory of Disordered Systems (Wiley, NY, 1988).

[7] F.M. Izrailev, S. Ruffo, and L.Tessieri, Journ. Phys. A: Math. Gen. 31, 5263 (1998).

[8] T.Kottos, G.P.Tsironis, and F.M.Izrailev, Journ. Phys. C., 9, 1777 (1997).

[9] J.M. Luck, Phys. Rev. B, 39, 5834 (1989).

[10] M. Griniasty and S. Fishman, Phys. Rev. Lett. 60, 1334 (1988).

[11] F.M. Izrailev and A.A. Krokhin, to be published.

[12] J.B. Sokoloff, Phys. Rep., 126189 (1985).

[13] R. Farchioni, G. Grosso, and G.P. Paravicini, Phys. Rev. B, 45, 6383 (1992).

[14] D.J. Thouless, Phys. Rev. Lett. 61, 2141 (1988).

[15] C.M. Soukoulis, M.J. Velgakis and E.N. Economou, Phys. Rev. B 505110 (1994).

[16] R. Johnson and B. Kramer, Z. Phys. B 63, 273 (1986).

[17] G.A. Korn and T.M. Korn, Mathematical Handbook, McGraw-Hill Book Company, Chapter 18.10, 1968.

[18] S. Das Sarma, Song He, and X.C. Xie, Phys. Rev. Lett. 61, 2144 (1988).

[19] R. Farchioni, G. Grosso, and G.P. Parravicini, J.Phys.: Condens. Matter 5, B13 (1993).

[20] F. Delyon, B. Simon, and B. Souillard, Phys. Rev. Lett. 52, 2187 (1984); Ann. Inst. Henri Poincarè, 42, 283 (1985).

[21] I. Guarneri, private communication.

[22] B.V. Chirikov, Phys. Reports 52, 263 (1979).

[23] P. Manneville and Y. Pomeau, Physica D, 1, 219 (1980).

[24] I. Goldhirsch, S.H. Noskowicz, and Z. Schuss, Phys. Rev. B. 47, 1918 (1992).

[25] F. de Moura and M.L. Lyra, Phys. Rev. Lett., 81, 3735 (1998). 